\title{
Conservative Circular Dichroism of an Extended Polypeptide Chain
}

\author{
Shoichi IKEDA \\ Department of Chemistry, Faculty of Science, Nagoya University, \\ Nagoya, Japan.
}

(Received May 1, 1972)

\begin{abstract}
Following Moffitt, Fitts, Kirkwood, and Tinoco, the dipole strength and rotatory strength associated with the transition to each exciton level of an excited state have been derived for an extended polypeptide chain of finite length, correct to the zeroth order approximation of wave functions. In terms of the nearest and the first two nearest neighbor approximations, sufficiently accurate for this polymer conformation, it is shown that light absorption and circular dichroism are induced essentially at two exciton levels alone, which are polarized parallel and perpendicularly, respectively. These two levels have rotatory strengths of equal magnitude but of opposite signs, for an electrically allowed transition. This is confirmed by application of the results to the $N-V_{1}$ transition of an extended polypeptide. The results are readily extended to an infinitely long extended polypeptide. From the comparison with the Loxsom's equation applied to the two-fold helix, it is pointed out that both the cyclic boundary condition and the Rosenfeld equation are valid in calculating rotatory properties of a long extended polypeptide. Generally, circular dichroism associated with an excited state can also be approximated by an equation expanded into a Taylor series, and the condition for its applicability is also indicated.

KEY WORDS Circular Dichroism / Chromophore / Exciton Level / Dipole Strength / Rotatory Strength / Transition Electric Dipole Moment / Transition Magnetic Dipole Moment / Ellipticity / Rotatory Oscillator Strength / Extended Polypeptide Chain /
\end{abstract}

The optical activity of helical polymers has been successfully treated by the Moffitt-Fitts -Kirkwood-Tinoco theory ${ }^{1-3}$ based on the Rosenfeld equation for rotatory strength. Total rotatory strength associated with an excited state of a polymer has been obtained to the first order approximation of polymer wave functions, which include interactions among chromophores in different excited states as well as in an identical excited state. ${ }^{3}$ In the original Moffitt's treatment ${ }^{1}$ which replaced the exciton coefficients of wave functions by means of the periodic boundary condition, an important term of optical rotation has been missed, which should appear even in the zeroth order approximation of polymer wave functions. Tinoco, Woody and Bradley ${ }^{4}$ pointed out that this term arises from the rotation exibited by the polymer molecules whose axes lie along the propagation direction of incident light. They derived equa- tions for rotatory disperson, valid to the zeroth order, and examined the effect of chain length. Tinoco ${ }^{5,6}$ further extended this theory to circular dichroism.

An extended polypeptide chain forms a linear alternating array of peptide and other chromophores, and it can be regarded as a two-fold helix, if the chromophores are arranged asymmetrically. This array itself acquires optical activity, if any one of polarization directions of the chromophore is neither parallel nor perpendicular to the chain direction and is skew with respect to it.

In the present paper, we will treat electronic properties of an extended polypeptide chain consisting of finite number of residues, confining ourselves to the zeroth order approximation of polymer wave functions, and derive expressions for dipole strength and rotatory strength associated with an excited state, both of which 
should be conservative. The treatment of rotatory properties will be finally extended to circular dichroism of an extended polypeptide chain of infinite length.

Recently, validity of the Rosenfeld equation was questioned when it was applied to helical polymers whose dimension exceeded the wavelength of incident light. ${ }^{7-9}$ Nevertheless, Loxsom $^{8}$ and Deutsche ${ }^{9}$ derived essentially identical expressions for optical rotation with Tinoco's equation, ${ }^{4}$ without relying on the Rosenfeld equation but based on different and more general approaches.

\section{FORMULATION}

The electronic Hamiltonian of a polymer chain composing of a "regular" array of $N$ identical residues is given by

$$
H=\sum_{k=1}^{N} \boldsymbol{H}_{k}+(1 / 2) \sum_{k=1}^{N} \sum_{l \neq k}^{N} V_{k, l}
$$

where $H_{k}$ is the Hamiltonian of the $k$-th residues in an isolated state but is assumed to be independent of its position, $k$, on the polymer chain. The second term, i.e., perturbation, is produced by the formation of "regular" chain configuration, and $V_{k, l}$ stands for Coulombic interaction between all electrons and of those with fixed nuclei within the two residues, $k$ and $l$.

If the real wave functions of an isolated $k$-th residue are $\phi_{k 0}$ and $\phi_{k \mathrm{e}}$ for the ground and an excited state, e, respectively, then the zeroth order polymer wave functions in the ground state and in the $K$-th exciton level of the excited state, E, are written as

$$
\phi_{0}^{0}=\prod_{k=1}^{N} \phi_{k 0}
$$

and

$$
\phi_{E K}^{0}=\sum_{k=1}^{N} C_{k \mathrm{e} K} \psi_{k \mathrm{e}}^{0}=\sum_{k=1}^{N} C_{k \mathrm{e} K} \phi_{k \mathrm{e}} \prod_{l \neq k}^{N} \phi_{l 0}
$$

As the exciton wave functions are normalized and orthogonal, it follows that

$$
\sum_{k=1}^{N} C_{k \ominus K} C_{k \ominus L}=\delta_{K, L}
$$

where $\delta_{K, L}$ is 1 for $K=L$ and 0 for $K \neq L$.

The first order energies of the polymer in the ground state and in the $K$-th exciton level of the excited state are calculated to be

$$
E_{0}^{1}=N E_{0}+(1 / 2) \sum_{k=1}^{N} \sum_{l \neq l}^{N} \int \phi_{0}^{0} V_{k, l} \psi_{0}^{0} \mathrm{~d} \tau
$$

and

$$
\begin{aligned}
E_{\mathrm{E} K}^{1}=E_{\mathrm{e}} & +(N-1) E_{0}+\sum_{k=1}^{N} C_{l \mathrm{e} K}^{2} \sum_{n \neq k}^{N} \int \phi_{k \mathrm{e}}^{0} V_{k, n} \psi_{k \mathrm{e}}^{0} \mathrm{~d} \tau \\
& +(1 / 2) \sum_{k=1}^{N} C_{k \mathrm{e} K}^{2} \sum_{m \neq k}^{N} \sum_{n \neq m}^{N} \int \psi_{m 0}^{0} V_{m, n} \psi_{n 0}^{0} \mathrm{~d} \tau \\
& +\sum_{k=1}^{N} \sum_{l \neq l e}^{N} C_{k \mathrm{e} K} C_{l \mathrm{e} K} \int^{n \neq k} \phi_{k \mathrm{e}}^{0} V_{k, l} \psi_{l \mathrm{e}}^{0} \mathrm{~d} \tau
\end{aligned}
$$

where $E_{0}$ and $E_{\mathrm{e}}$ are the energies of an isolated residue in the ground state and the excited state, respectively. Both the second term of eq 5 and the fourth term of eq 6 are (weighted sums of) interactions among residues all in the ground state and thus represent permanent dipole-permanent dipole interactions in the dipole approximation. The third term of eq 6 is the interactions of a single excited residue with all the other residues in the ground state. Those electrostatic terms shift the center of gravity of energy levels relative to the zeroth order energies.

If the first order perturbation energies of the polymer are measured from the states in which each residue is put in the electrostatic field of the rest of the polymer, the first order energies of the polymer become

and

$$
E_{0}^{1}=N E_{0}^{0}
$$

$E_{\mathrm{E} K}^{1}=E_{\mathrm{e}}^{0}+(N-1) E_{0}^{0}+\sum_{k=1}^{N} \sum_{l \neq l}^{N} C_{l \mathrm{e} K} C_{l \mathrm{e} K} V_{k 0 \mathrm{e}, l 0 \mathrm{e}}$

where $E_{0}^{0}$ and $E_{\mathrm{e}}^{0}$ are the zeroth order energies of the polymer in the ground and the excited states, respectively, and may be referred to as the unperturbed energies. The exchange interaction is

$$
\begin{aligned}
V_{l k 0 \mathrm{e}, l 0 \mathrm{e}} & =\int \phi_{l \mathrm{e}}^{0} V_{k, l} \phi_{l \mathrm{e}}^{0} \mathrm{~d} \tau \\
& =\iint \phi_{k \mathrm{e}} \phi_{l 0} V_{k, l} \phi_{k 0} \phi_{l \mathrm{e}} \mathrm{d} \tau_{k} \mathrm{~d} \tau_{l}
\end{aligned}
$$

As the center of gravity of exciton levels in the excited state should be coincident with the unperturbed level, it follows, together with eq 4 , that

$$
\sum_{K=1}^{N} C_{k \mathrm{e} K} C_{l \mathrm{e} K}=\delta_{k, l}
$$


which will be utilized in Appendix. Then the frequency for the $K$-th exciton level of the excited state is given by

$$
\nu_{\mathrm{E} K}=\nu_{\mathrm{EO}}+(1 / h) \sum_{k=1}^{N} \sum_{l \neq l k}^{N} C_{k \mathrm{e} K} C_{l \mathrm{e} K} V_{k 0 \mathrm{e}, l 0 e}
$$

where $h$ is Planck's constant and $\nu_{\mathrm{EO}}=\left(E_{\mathrm{e}}^{0}-E_{0}^{0}\right) / h$ is the excitation frequency of a residue in the static field of the rest of the polymer. The exciton coefficients, $C_{l e \mathrm{~K}}$, can be obtained by solving a set of equations

$$
\sum_{k=1}^{N} C_{k \mathrm{e} K}\left(V_{k 00, l 0 \mathrm{e}}-\delta_{k, l} E_{\mathrm{E} K}^{\prime}\right)=0 \quad l=1,2, \ldots, N
$$

for each level, $K$, or $E_{\mathrm{E} K}^{\prime}$, where $E_{\mathrm{E} K}^{\prime}=E_{\mathrm{E} K}^{1}-E_{0}^{1}$ $-\left(E_{\mathrm{e}}^{0}-E_{0}\right)$, together with eq 4.

The dipole strength of the $K$-th level is then expressed by

$$
\begin{aligned}
D_{\mathrm{E} K} & =\left(\int \phi_{0}^{0}\left(\sum_{k=1}^{N} \boldsymbol{\mu}_{k}\right) \psi_{\mathrm{E} K}^{0} \mathrm{~d} \tau\right) \cdot\left(\int \psi_{0}^{0}\left(\sum_{k=1}^{N} \boldsymbol{\mu}_{k}\right) \psi_{\mathrm{E} K}^{0} \mathrm{~d} \tau\right) \\
& \left.=\left(\boldsymbol{\mu}_{0 \mathrm{e}} \cdot \boldsymbol{\mu}_{0 \mathrm{e}}\right)+\sum_{k=1}^{N} \sum_{l \neq k}^{N} C_{k \mathrm{e} K} C_{l \mathrm{e} K}\left(\boldsymbol{\mu}_{k 0 \mathrm{e}} \cdot \boldsymbol{\mu}_{l 0 \mathrm{e}}^{l}\right)\right)(13)
\end{aligned}
$$

and, by means of the Rosenfeld equation, the corresponding rotatory strength is calculated as

$$
\begin{aligned}
\boldsymbol{R}_{\mathrm{E} K}= & \operatorname{Im}\left(\int \phi_{0}^{0}\left(\sum_{k=1}^{N} \boldsymbol{\mu}_{k}\right) \psi_{\mathrm{E} K}^{0} \mathrm{~d} \tau\right) \\
& \cdot\left(\int \phi_{\mathrm{E} K}^{0} \sum_{k=1}^{N}\left\{\mathbf{m}_{k}+i \frac{\pi \nu_{\mathrm{EO}}}{c}\left[\mathbf{R}_{k} \times \boldsymbol{\mu}_{k}\right]\right\} \psi_{0}^{0} \mathrm{~d} \tau\right) \\
= & \operatorname{Im}\left\{\left(\boldsymbol{\mu}_{0 \mathrm{e}} \cdot \mathbf{m}_{\mathrm{e} 0}\right)+\sum_{k=1}^{N} \sum_{l \neq k}^{N} C_{k \mathrm{e} K} C_{l \mathrm{e} K}\left(\boldsymbol{\mu}_{k 0 \mathrm{e}} \cdot \mathbf{m}_{l \mathrm{e} 0}\right)\right\} \\
& -\left(\pi \nu_{\mathrm{EO}} / 2 c\right) \sum_{k=1}^{N} \sum_{l \neq k}^{N} C_{k \mathrm{e} K} C_{l \mathrm{e} K} \\
& \times\left\{\left(\mathbf{R}_{l}-\mathbf{R}_{k}\right) \cdot\left[\boldsymbol{\mu}_{k 0 \mathrm{e}} \times \boldsymbol{\mu}_{l 0 \mathrm{e}}\right]\right\}
\end{aligned}
$$

where $c$ is the velocity of light. Here it is assumed that the $k$-th residue has a chromophore at a position, $\mathbf{R}_{k}$, measured from an arbitrary common origin, and that the chromophore is characterized by the transition electric dipole moment

$$
\boldsymbol{\mu}_{k 0 \mathrm{e}}=\int \phi_{0}^{0} \boldsymbol{\mu}_{k} \psi_{k \mathrm{e}}^{0} \mathrm{~d} \tau=\int \phi_{k 0} \mu_{k} \phi_{k \mathrm{e}} \mathrm{d} \tau_{k}
$$

and the transition magnetic dipole moment around the center of the chromophore

$$
\mathbf{m}_{k \mathrm{e} 0}=\int \phi_{k \mathrm{e}}^{0} \mathbf{m}_{k} \psi_{0} \mathrm{~d} \tau=\int \phi_{k \mathrm{e}} \mathbf{m}_{k} \phi_{k 0} \mathrm{~d} \tau_{k}
$$

both at the frequency, $\nu_{E O}$, where the operator $\boldsymbol{\mu}_{k}$ is the sum of the charge times position vectors over all electrons and nuclei within the $k$-th chromophore, and the operator $\mathbf{m}_{k}$ is the corresponding sum of angular momentum vectors around the center of the chromophore, multiplied by the magnetogyric ratio. In an electrically allowed transition, the magnetic moment terms are small and the last term of eq. 14, i.e., the exciton coupled oscillator term, is important.

In the zeroth-order approximation of polymer wave functions, both the total dipole strength and the total rotatory strength for the excited state, E, are conservative:

$$
\sum_{K=1}^{N} D_{\mathrm{E} K}=N \mu_{0 \mathrm{e}}^{2}
$$

and

$$
\sum_{K=1}^{N} R_{\mathrm{E} K}=N \operatorname{Im}\left(\mu_{0 \mathrm{e}} \cdot \mathbf{m}_{\mathrm{e} 0}\right)
$$

Residue extinction coefficient, $\varepsilon\left(1 \mathrm{~cm}^{-1} \mathrm{~mol}^{-1}\right)$, and residue ellipticity, $[\theta]\left(\right.$ degree $\mathrm{cm}^{2}$ decimol $^{-1}$ ), for the $K$-th exciton level of the excited state, $\mathrm{E}$, are given by

and

$$
\varepsilon_{\mathrm{E} K}=\left(8 \pi^{3} N_{\mathrm{A}} / 6909 h c N\right) \nu D_{\mathrm{E} K} f\left(\nu-\nu_{\mathrm{E} K}\right)
$$

$$
[\theta]_{\mathrm{E} K}=\left(48 \pi^{2} N_{\mathrm{A}} / h c N\right) \nu R_{\mathrm{E} K} f\left(\nu-\nu_{\mathrm{E} K}\right)
$$

at a frequency, $\nu$, respectively, where $N_{\mathrm{A}}$ is Avogadro's number and $f\left(\nu-\nu_{E K}\right)$ is the spectral shape function, positive and normalized. (The spectral shape function may also be defined by a function into which the factor, $\nu$, in eq 19 and 20 , but replaced by $\nu_{E K}$, is incorporated, in the case of infinitely long polymers. ${ }^{5}$ )

Total residue ellipticity for the excited state, $\mathrm{E}$, is then obtained by summing eq 20 over all exciton levels:

$$
[\theta]_{\mathrm{E}}=\left(48 \pi^{2} N_{\mathrm{A}} / h c N\right) \nu \sum_{K=1}^{N} R_{\mathrm{E} K} f\left(\nu-\nu_{\mathrm{E} K}\right)
$$

Each spectral shape function at a frequency, $\nu$, can be approximated by a Taylor expansion with respect to $\nu-\nu_{E K}$ around $\nu-\nu_{E O}$, provided that $\left|\nu_{\mathrm{E} K}-\nu_{\mathrm{EO}}\right|$ is small. Then

$$
f\left(\nu-\nu_{E K}\right)=f\left(\nu-\nu_{E O}\right)-\left(\nu_{E_{K}}-\nu_{E O}\right) \frac{\partial f\left(\nu-\nu_{E O}\right)}{\partial \nu}
$$


If the spectral shape function is common to all the levels, eq 21 can be approximately represented by

$$
\begin{aligned}
{[\theta]_{\mathrm{E}}=} & \frac{48 \pi^{2} N_{\mathrm{A}}}{h c N} \nu\left\{\left(\sum_{K=1}^{N} R_{\mathrm{E} K}\right) f\left(\nu-\nu_{\mathrm{EO}}\right)\right. \\
& \left.\left.-\left(\sum_{K=1}^{N}\left(\nu_{\mathrm{E} K}-\nu_{\mathrm{EO}}\right) R_{\mathrm{E} K}\right)\right) \frac{\partial f\left(\nu-\nu_{\mathrm{EO}}\right)}{\partial \nu}\right\}
\end{aligned}
$$

The first term gives a normal ellipticity band with the rotatory strength, $\sum R_{\mathrm{E} K}$, which is equal to the intrinsic rotatory strength to the zeroth order approximation (eq 18). The second term produces a pair of positive and negative ellipticity bands, and their sign and strength are determined by the total rotatory oscillator strength, $\sum \nu_{E_{K}} R_{\mathrm{E} K}$, as well as by the total rotatory strength. As shown in Appendix, this is estimated as

$$
\begin{aligned}
& \sum_{K=1}^{N}\left(\nu_{\mathrm{E} K}-\nu_{\mathrm{EO}}\right) R_{\mathrm{E} K} \\
&=(1 / h) \operatorname{Im}\left\{\sum_{k=1}^{N} \sum_{l \neq k}^{N}\left(\boldsymbol{\mu}_{k 0 \mathrm{e}} \cdot \mathbf{m}_{l \mathrm{e} 0}\right) V_{k 0 \mathrm{e}, l 0 \mathrm{e}}\right\} \\
&-\left(\pi \nu_{\mathrm{EO}} / 2 h c\right) \sum_{k=1}^{N} \sum_{l \neq k}^{N}\left(( \mathbf { R } _ { l } - \mathbf { R } _ { k } ) \cdot \left(\boldsymbol{\mu}_{k 0 \mathrm{e}}\right.\right. \\
&\left.\left.\times \boldsymbol{\mu}_{l 0 \mathrm{e}}\right]\right) V_{k 0 \mathrm{e}, l 0 \mathrm{e}}
\end{aligned}
$$

Equation 23 is valid to the first order, if the total rotatory strength is evaluated to the first order $^{3}$ rather than by eq 18 . Thus eq 23 has more general applicability than eq $21 .^{6}$

All the above equations are applicable to oligomers of any rigid configuration, as far as all the residues are identical.

\section{GEOMETRY OF AN EXTENDED POLYPEPTIDE CHAIN}

Let us choose a right-handed rectangular coordinate on an extended polypeptide chain, as shown in Figure 1. The $y$-axis $\left(\mathbf{e}_{2}\right)$ directs parallel to the chain direction through its center line, and the $x$ - and $z$-axes $\left(\mathbf{e}_{1}\right.$ and $\mathbf{e}_{3}$ ) are arbitrarily assigned to the directions of $\mathrm{N}-\mathrm{H}$ or $\mathrm{C}=\mathrm{O}$ bonds and side chains, respectively. A reference point specifying position of the $k$-th residue is defined on the center line as $\mathbf{e}_{2} k b$, where $b$ is the repeat distance of residues along the chain direction, and its chromophore is attached to a position, $\mathbf{e}_{2} q+(-1)^{k}\left(\mathbf{e}_{1} a \sin \phi+\right.$ $\mathbf{e}_{3} a \cos \phi$ ), relative to the reference point, where $a$ is the distance of a chromophore from the center line and $\phi$ is the azimuthal angle of perpendicular projection of position vector of a chromophore making with the $z$-axis, as measured toward the $x$-axis. The position of the $k$-th chromophore is then given by

$$
\mathbf{R}_{k}=\mathbf{e}_{2}(k b+q)+(-1)^{k} a\left(\mathbf{e}_{1} \sin \phi+\mathbf{e}_{3} \cos \phi\right)
$$

If we imagine a circumscribed cylinder at the chromophore positions and define the tangential $(\mathrm{t})$, parallel $(/ /)$ and radial $(\mathrm{r})$ components of each transition dipole as in the right-handed $x y z$ coordinate, where the parallel component is always taken in the direction of $y$-axis $\left(\mathbf{e}_{2}\right)$, then the transition moments can be expressed by

$$
\begin{aligned}
\boldsymbol{\mu}_{k 0 \mathrm{e}}=\mathbf{e}_{2} \mu_{0 \mathrm{e}, / /} & +(-1)^{k}\left[\mathbf{e}_{1}\left(\mu_{0 \mathrm{e}, r} \sin \phi+\mu_{0 \mathrm{e}, t} \cos \phi\right)\right. \\
& +\mathbf{e}_{3}\left(\mu_{0 \mathrm{e}, r} \cos \phi-\mu_{\mathrm{ce}, t} \sin \phi\right]
\end{aligned}
$$

for the electric dipole and by a similar expression for the magnetic dipole.

The interaction potential between two chromophores, $k$ and $l$, for an identical transition, $0-e$, can be calculated by the dipole approximation:

$$
\begin{aligned}
V_{k 0 \mathrm{e}, l 0 \mathrm{e}}= & \left\{\mu_{0 \mathrm{e}, \|}^{2}+(-1)^{k+l} \mu_{0 \mathrm{e}, \perp}^{2}\right. \\
- & \left.\frac{3\left\{-2\left[1-(-1)^{k+l}\right] a^{2} \mu_{0 \mathrm{e}, r}^{2}+(l-k)^{2} b^{2} \mu_{0 \mathrm{e}, ~}^{2}\right\}}{2\left[1-(-1)^{k+l}\right] a^{2}+(l-k)^{2} b^{2}}\right\} \\
& /\left\{2\left[1-(-1)^{k+l}\right] a^{2}+(l-k)^{2} b^{2}\right\}^{3 / 2}
\end{aligned}
$$

where $\mu_{0 \mathrm{e}, \perp}=\sqrt{\mu_{0 \mathrm{e}, t}^{2}+\mu_{0 \mathrm{e}, r}^{2}}$ is the perpendicular component of transition electric moment, and the effective dielectric constant is, as usual,
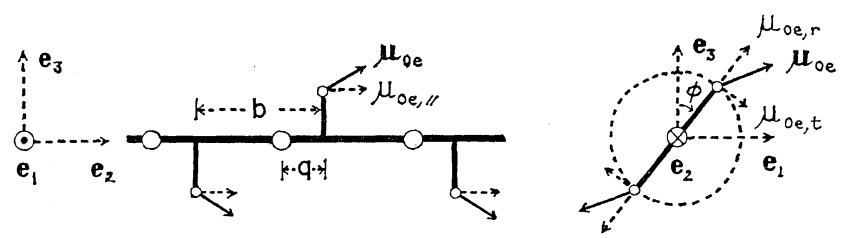

Figure 1. Geometry of a linear alternating array of chromophores: $\bigcirc$, center of residue; $O$, center of chromophore, $\otimes, \odot,-$, center line; $/$, side chain. 
assumed to be unity. A more accurate monopole approximation for the interaction potential requires quantum mechanical calculation of electronic structure of the chromophore. If a transition monopole of the $k$-th residue has a charge, $\rho_{k 0 \mathrm{e}, \sigma}$, at a position, $\mathbf{R}_{k, \sigma}$, the interaction potential is given by

$$
V_{k 0 \mathrm{e},, l 0 \mathrm{e}}=\sum_{k=1}^{N} \sum_{\sigma} \rho_{k 0 \mathrm{e}, \sigma} \sum_{l \neq k}^{N} \sum_{\tau} \rho_{l 0 \mathrm{e}, \tau} /\left|\left(\mathbf{R}_{l, \tau}-\mathbf{R}_{k, \sigma}\right)\right|
$$

\section{SPECTRAL STRENGTHS AND CIRCULAR DICHROISM OF AN EXTENDED OLIGOPEPTIDE CHAIN}

Dipole strength and rotatory strength of an extended polypeptide chain for the transition to the $K$-th level of an excited state, E, are written in the form

$$
D_{\mathrm{E} K}=\boldsymbol{P}_{K} \mu_{0 \theta, / /}^{2}+Q_{K} \mu_{0 \mathrm{e}, \perp}^{2}
$$

and

$$
\begin{aligned}
R_{\mathrm{E} K}= & \operatorname{Im}\left\{\boldsymbol{P}_{K}\left(\mu_{0 \mathrm{e}, / /} m_{\mathrm{e} 0, \|}\right)+\boldsymbol{Q}_{K}\left(\mu_{0 \mathrm{e}, \perp} m_{\mathrm{e} 0, \perp}\right)\right\} \\
& +\left(\pi \nu_{\mathrm{EO}} / \boldsymbol{c}\right)\left(\boldsymbol{P}_{K}-Q_{K}\right) a \mu_{0 \mathrm{e}, t} \mu_{0 \mathrm{e}, \|}
\end{aligned}
$$

where

$$
\begin{aligned}
& P_{K}=\sum_{k=1}^{N} \sum_{l \neq k}^{N} C_{k \mathrm{e} K} C_{l \mathrm{e} K}+1 \\
& Q_{K}=\sum_{\substack{k=1 \\
l \neq 1}}^{N} \sum_{\substack{l \neq k \\
l \neq l}}^{N} C_{k \mathrm{e} K} C_{l \mathrm{e} K}-\sum_{\substack{l k=1 \\
l \\
k+l: l: \text { odd }}}^{N} \sum_{\substack{l \neq k \\
\text { oven }}}^{N} C_{k \mathrm{e} K} C_{l \mathrm{e} K}+1
\end{aligned}
$$

The last term of eq 30 , i.e.,

$$
\left(2 \pi \nu_{\mathrm{EO}} / c\right)\left(\sum_{\substack{k=1 \\ k+l: \text { odd }}}^{N} \sum_{\substack{l \neq k \\ N}}^{N} C_{k \mathrm{e} K} C_{l \mathrm{e} K}\right) a \mu_{0 \mathrm{e}, t} \mu_{0 \mathrm{e}, \|} \text {, is the ex- }
$$

citon coupled oscillator term making major contribution in an electrically allowed transition, and its form shows that the presence of chro- mophores on the opposite sides along an extended chain are responsible for the rotatory power.

Residue ellipticity for the excited state, E, can be expressed by eq 23 , in which the total rotatory strength is given by eq 18 and the rotatory oscillator strength term is calculated by the use of eq 24 as

$$
\begin{aligned}
& \sum_{K=1}^{N}\left(\nu_{\mathrm{E} K}-\nu_{\mathrm{EO}}\right) R_{\mathrm{E} K} \\
& =(1 / h) \operatorname{Im}\left(\mu_{0 \mathrm{e}, ~} m_{\mathrm{e} 0, \|}\right)\left(\sum_{k=1}^{N} \sum_{l \neq k}^{N} V_{k 0 \mathrm{e}, l 0 \mathrm{e}}\right) \\
& +(1 / h) \operatorname{Im}\left(\mu_{0 \mathrm{e}, \perp}\right)\left(\sum_{\substack{k=1 \\
k+l=1 \\
k: \text { even }}}^{N} \sum^{N} V_{k 0 \mathrm{e}, l 0 \mathrm{e}}\right. \\
& \left.-\sum_{\substack{k=1 \\
k+1: \text { odd }}}^{N} \sum_{\substack{l \neq k \\
k+k}}^{N} V_{k 0 \mathrm{e}, l 0 \mathrm{e}}\right) \\
& +\left(2 \pi \nu_{\mathrm{EO}} / h c\right)\left(a \mu_{0 \mathrm{e}, t} \mu_{0 \mathrm{e}, \| /}\right)\left(\sum_{\substack{k=1 \\
k+l: \text { odd }}}^{N} \sum_{\substack{l \neq k \\
\prime}}^{N} V_{k 0 \mathrm{e}, l 0 \mathrm{e}}\right)
\end{aligned}
$$

\section{Symmetry Considerations}

For polypeptides consisting of even number of residues, the polymer wave functions, $\phi_{k \mathrm{e}}^{0}$, which obtain in the absence of perturbation, belong to the symmetry group, $\mathbf{S}_{2}$, and the zeroth-order polymer wave functions, $\psi_{\mathrm{E}_{K}}^{0}$, of an excited state can be classified into the two types, those of the representations, $A_{1 \mathrm{~g}}$ and $A_{\mathrm{iu}}$. The $A_{1 \mathrm{~g}}$ levels are expressed by the wave functions with the exciton coefficients, $C_{k \mathrm{e} K}=C_{(N-k) \mathrm{e} K}$, and the $A_{1 \mathrm{u}}$ levels have the wave functions with the coefficients, $C_{k \mathrm{e} K}=-C_{(N-k) \mathrm{e} K}$.

For polypeptides with odd number of residues, the wave functions, $\psi_{k \mathrm{e}}^{0}$, belong to the symmetry group, $\mathrm{C}_{2 \mathrm{~V}}$, and the zeroth order polymer wave

Table I. Expressions for the coefficients, $P_{K}$ and $Q_{K}$, of eq 31 as derived from the symmetry considerations

\begin{tabular}{lcccc}
\hline$N$ & Symmetry & $K$ & $P_{K}$ & $Q_{K}$ \\
\hline \multirow{2}{*}{ Even } & $\mathrm{S}_{2}$ & $A_{\mathrm{gg}}$ & $4\left(\sum_{k=1}^{N / 2} C_{k \mathrm{e} K)^{2}}\right.$ & 0 \\
& & $A_{\mathrm{uu}}$ & 0 & $4\left(\sum_{k=1}^{N / 2}(-1)^{k} C_{k \mathrm{e} K)^{2}}\right.$ \\
\hline \multirow{2}{*}{ Odd } & $\mathrm{C}_{2 v}$ & $A_{1}$ & $4\left(\sum_{k=1}^{N-1 / 2} C_{k \mathrm{e} K}\right)\left(\sum_{k=1}^{N+1 / 2} C_{k \mathrm{e} K}\right)+C^{2}{ }_{N+1 / 2, \mathrm{e} K}$ & $4\left(\sum_{k=1}^{N-1 / 2}(-1)^{k} C_{k \mathrm{e} K}\right)\left(\sum_{k=1}^{N+1 / 2}(-1)^{k} C_{k \mathrm{e} K}\right)+C^{2} N+1 / 2, \mathrm{e} K$ \\
& & $B_{1}$ & 0 & 0 \\
\hline
\end{tabular}


functions, $\phi_{\mathrm{E}_{K}}^{0}$, can be divided into the two, one being of $A_{1}$ and the other of $B_{1}$. The $A_{1}$ levels are characterized by the coefficients, $C_{k \in K}=C_{(N-k) \Theta K}$, and the $B_{1}$ levels are by $C_{k \mathrm{e} K}=-C_{(N-k)}$ and $C_{((N+1) / 2) \Theta K}=0$.

The dipole strength and rotatory strength are given by eq 29 and 30, in which the coefficients are now partly simplified as tabulated in Table I. It can be seen that the exciton levels of evenmembered polypeptides are divided into two, one $\left(A_{1 \mathrm{~g}}\right)$ being polarized parallel and the other being perpendicular, both of which are optically active. In odd-membered polypeptides, half of the exciton levels, i.e., the $B_{1}$ levels, do not contribute to light absorption nor optical rotation.

\section{Neighbor Approximations}

Since no further reduction is possible from symmetry considerations, we will introduce an approximate method by means of physical considerations.

For an extended polypeptide chain, it is evident that the largest interaction potential should occur at the nearest or the second nearest neighbors, depending on whether the chromophores are closer to the center line or far apart from it, as compared with the repeat distance, that is, roughly, on $\mathbf{a}<\mathbf{b}$ or $\mathbf{a}>\mathbf{b}$. We can then anticipate that the nearest or the first two neighbor approximation would be a sufficiently good approach for an extended polypeptide chain. It might be better to include the interactions with the second neighbors for the former case and those with the fourth neighbors for the latter case. However, those additional interactions would not be concerned with the exciton coupled oscillator term of rotatory strength, since this term is related only with the interactions of excited chromophores with those on the opposite side along the chain. Furthermore, the following mathematical device for neighbor approximations makes the exciton coefficients independent of the magnitude of interaction potentials between any pair.

Bradley, Tinoco and Woody ${ }^{10}$ have solved the perturbation problem, eq 12 , by means of the nearest neighbor approximation, i.e.,

$$
V_{k 00, l 0 \mathrm{e}}=0 \text { unless } l=k-1 \text { or } k+1
$$

Later, Bradley, Lifson, and Honig ${ }^{11}$ observed that the problem can also be solved analytically even to the first two neighbor approximation, i.e.,

$$
V_{k 0 \mathrm{e}, l 0 \mathrm{e}}=0 \text { unless } l=k-2, k-1, k+1 \text { or } k+2
$$

if the upper-left and the lower-right corner terms of eq 12, i.e., the coefficients of $C_{1 \mathrm{e} K}$ for $l=1$ and of $C_{N e K}$ for $l=N$, are replaced by $-V_{10 \mathrm{e}, 30 \mathrm{e}}$ $-E_{\mathrm{E} K}^{\prime}$. In both approximations, the exciton coefficients have an identical expression

$$
C_{k \mathrm{e} K}=\sqrt{2 /(N+1)} \sin (\pi k K /(N+1))
$$

In terms of those exciton coefficients, spectral strengths can be calculated after Bradley, Tinoco and Woody. ${ }^{10}$ The final expressions can be written in the form of eq 29 and 30, in which the coefficients, $P_{K}$ and $Q_{K}$, are those given in Table II.

Table II. Expressions for the coefficients, $\boldsymbol{P}_{K}$ and $Q_{K}$, of eq 31 in the neighbor approximations

\begin{tabular}{cccc}
\hline$N$ & $K$ & $P_{K}$ & $Q_{K}$ \\
\hline \multirow{2}{*}{ Even } & Odd & $\frac{2}{N+1} \cot ^{2} \frac{\pi K}{2(N+1)}$ & 0 \\
& Even & 0 & $\frac{2}{N+1} \tan ^{2} \frac{\pi K}{2(N+1)}$ \\
\hline \multirow{2}{*}{ Odd } & Odd & $\frac{2}{N+1} \cot ^{2} \frac{\pi K}{2(N+1)}$ & $\frac{2}{N+1} \tan ^{2} \frac{\pi K}{2(N+1)}$ \\
& Even & 0 & 0
\end{tabular}

Because of the properties of cot and tan functions, we see that only two among $N$ levels have significant spectral strengths and all the other levels have much weaker or negligibly small light absorption and optical rotation. The maximum strengths are then approximately expressed by

$$
\begin{aligned}
& D_{\mathrm{E}_{1}}=(2 /(N+1)) \mu_{0 \mathrm{e}, / /}^{2} \cot ^{2}(\pi / 2(N+1)) \\
& D_{\mathrm{E} N}=(2 /(N+1)) \mu_{0 \mathrm{e}, \perp}^{2} \cot ^{2}(\pi / 2(N+1))
\end{aligned}
$$

and

$$
\begin{aligned}
R_{\mathrm{E} 1}= & (2 /(N+1))\left\{\operatorname{Im}\left(\mu_{0 \mathrm{e}, \|} m_{\mathrm{e} 0, \|}\right)\right. \\
& \left.+\left(\pi \nu_{\mathrm{EO}} / c\right) a \mu_{0 \mathrm{e}, t} \mu_{0 \mathrm{e}, \|}\right\} \cot ^{2}(\pi / 2(N+1)) \\
R_{\mathrm{E} N}= & (2 /(N+1))\left\{\operatorname{Im}\left(\mu_{0 \mathrm{e}, \perp} m_{\mathrm{e}_{0}, \perp}\right)\right. \\
& \left.-\left(\pi \nu_{\mathrm{EO}} / c\right) a \mu_{0 \mathrm{e}, t} \mu_{0 \mathrm{e}, \|}\right\} \cot ^{2}(\pi / 2(N+1))
\end{aligned}
$$

From these results we may conclude that an extended polypeptide chain of finite length has 


\section{S. IKEDA}

essentially two levels, which exhibit strong absorption and are polarized parallel and perpendicularly, respectively. Furthermore, in an electrically allowed transition, only those two levels are strongly optically active and form a pair of positive and negative ellipticity bands, with equal magnitude. Thus eq 21 can be written as

$$
\begin{aligned}
{[\theta]_{\mathrm{E}}=\left(48 \pi^{2} N_{\mathrm{A}} / h c N\right) } & \nu\left\{R_{\mathrm{E}_{1}} f\left(\nu-\nu_{\mathrm{E}_{1}}\right)\right. \\
& \left.+R_{\mathrm{E}_{N}} f\left(\nu-\nu_{\mathrm{E}_{N}}\right)\right\}
\end{aligned}
$$

where $\nu_{\mathrm{E}_{1}}$ and $\nu_{\mathrm{E} N}$ are the frequencies of parallel and perpendicular levels. Those frequencies are given by equations, appreciably dependent on the approximation used, but can be exactly evaluated by eq 11 with the exact exciton coefficients.

\section{CIRCULAR DICHROISM OF AN EXTENDED POLYPEPTIDE CHAIN OF INFINITE LENGTH}

\section{Unoriented Polypeptide Chain}

For a long helical or "regular" polymer, the exciton levels of an excited state are distributed continuously over a wide frequency region. Among those, only two levels contribute to light absorption, which are polarized paralle and perpendicularly, respectively. According to the Moffitt-Fitts-Kirkwood-Tinoco theory, however, all these levels are optically active. Specifically, for a long extended polymer, the neighbor approximations which were applied for an extended oligopeptide are expected to hold as well. Then all the equations derived for an extended oligopeptide chain should be valid even for a very long extended chain. This leads to the results that essentially only two levels, polarized parallel and perpendicularly, respectively, are optically active and that circular dichroism of a long extended polypeptide chain will be represented by a twoterm equation such as eq 36.

Recently, Loxsom ${ }^{8}$ has derived equations of optical rotation for an infinitely long helical polymer, based on the Stephen's equation. The results can be readily applied to an extended polypeptide chain, which is regarded as a twofold helix. Transformation of optical rotation into circular dichroism can be carried out after
Moffitt and Moscowitz. ${ }^{12}$ The frequency is expressed by a complex frequency, $\nu+i \varepsilon$, and the equation for optical rotation is converted into a complex form by the identity, $\left(x-i \varepsilon^{-1}\right)=$ $x^{-1}+i \pi \delta(x)$, where $\varepsilon$ is a small positive quantity and $\delta(x)$ is the Dirac $\delta$ function. ${ }^{13}$ Then its real part is residue rotation and its imaginary part is residue ellipticity, after $\delta(x)$ 's with large $x$ values have been ignored and $\delta\left(\nu-\nu_{E_{K}}\right)$ has been replaced by the spectral shape, $f\left(\nu-\nu_{E K}\right)$.

The final equation for residue ellipticity of an extended polypeptide chain randomly oriented against incident light is given by

$$
\begin{aligned}
{[\theta]_{\mathrm{E}}=\left(48 \pi^{2} N_{\mathrm{A}} / h c N\right) \nu\left\{\boldsymbol{R}_{\mathrm{E}} /\left(f \nu-\nu_{\mathrm{E} / l}\right)\right.} \\
\left.+R_{\mathrm{E} \perp} f\left(\nu-\nu_{\mathrm{E} \perp}\right)\right\}
\end{aligned}
$$

where the frequencies of parallel and perpendicular transitions are

$$
\begin{aligned}
& \nu_{\mathrm{E} / /}=\nu_{\mathrm{EO}}+(1 / N h) \sum_{k=1}^{N} \sum_{l \neq k}^{N} V_{k 0 \mathrm{e}, l 0 \mathrm{e}} \\
& \nu_{\mathrm{E} \perp}=\nu_{\mathrm{EO}}+(1 / N h)\left(\sum_{\substack{k=1 \\
k+l: \text { even }}}^{N} \sum_{\substack{l \neq k \\
k+l}}^{N} V_{k 0 \mathrm{e}, i 0 \mathrm{e}}-\sum_{\substack{k=1 \\
l}}^{N} \sum_{\substack{l \neq k \\
l+l: \text { odd }}}^{N} V_{l k 0 \mathrm{e}, l 0 \mathrm{e}}\right)
\end{aligned}
$$

and the associated rotatory strengths are, correct to the zeroth order,

$$
\begin{aligned}
& R_{\mathrm{E} \|}=N\left\{\operatorname{Im}\left(\mu_{0 \mathrm{e}, \|} m_{\mathrm{e} 0, \|}\right)+\left(\pi \nu_{\mathrm{EO}} / c\right) a \mu_{0 \mathrm{e}, t} \mu_{0 \mathrm{e}, \|}\right\} \\
& R_{\mathrm{E} \perp}=N\left\{\operatorname{Im}\left(\mu_{0 \mathrm{e}, \perp} m_{\mathrm{e} 0, \perp}\right)-\left(\pi \nu_{\mathrm{EO}} / c\right) a \mu_{0 \mathrm{e}, t} \mu_{0 \mathrm{e}, \|}\right\} .
\end{aligned}
$$

Equation 37 indicates that only the two exciton levels which are polarized parallel and perpendicularly, respectively, are optically active in a long extended polypeptide chain. As seen from eq 36, this has also been satisfied, to a good approximation, by an extended oligopeptide chain. In particular, the two rotatory strengths of an infinitely long extended polypeptide are completely identical with those of an extended dipeptide, although their locations, which are related to the first order approximation, are different. Equation 39 suggests that each of the two rotatory strengths is simply a sum of the intrinsic strength of a chromophore itself and the strength arising from the shift of a residue center from the chromophore position to the chain axis.

Equation 37 can be approximated by eq 23, as far as the two levels are close to the unperturbed level. 


\section{Oriented Polypeptide Chain}

Loxsom $^{8}$ has also derived the equation of optical rotation for an infinitely long helical polymer, whose axis is parallel to the direction of propagation of incident light. For a long extended polypeptide it can be transformed into the equation of ellipticity, which can be written as

$$
[\theta]_{\mathrm{E}}{ }^{\prime \prime}=\left(72 \pi^{2} N_{\mathrm{A}} / h c N\right) \nu\left\{\operatorname{Im}\left(\mu_{0 \mathrm{e}, \perp} m_{\mathrm{e} 0, \perp}\right)\right\} f\left(\nu-\nu_{\mathrm{E} \perp}\right)
$$

Equation 40 shows that a linear alternating stack of chromophores is optically inactive for light incident parallel to the array, unless the chromophore itself is optically active.

It has been demonstrated ${ }^{4.5 .7 .9}$ that characteristic paired ellipticity bands should be observed in circular dichroism of a long general helix when the incident light impinges parallel to the helix axis. However, eq 40 indicates that such rotatory power cannot be exhibited by a twofold helix. This suggests that both the cyclic boundary condition and the Rosenfeld equation are applicable in deriving rotatory properties of a long extended polypeptide chain, since the original Moffitt's error ${ }^{1}$ in the calculation of rotatory power of general helices has been ascribed to his missing the contribution from the helices lying parallel to the incident light, ${ }^{4}$ which should have come from his use of the periodic boundary condition, after Moffitt, Fitts and Kirkwood ${ }^{2}$ and Tinoco, ${ }^{3.4}$ but which should have arisen from his use of the Rosenfeld equation, after Ando, ${ }^{7}$ Loxsom, ${ }^{8}$ and Deutsche. ${ }^{9}$

\section{SIGN OF ELLIPTICITY BANDS FOR AN ELECTRICALLY ALLOWED TRANSITION}

The equations derived for $R_{\mathrm{E} / /}$ and $R_{\mathrm{E} \perp}$ (eq 30 or 35 and 39) contain the factor, $\mu_{0 \mathrm{e}, t} \mu_{0 \mathrm{e}, / /}$, which determines the sign of a pair of ellipticity bands for an electrically allowed transition, that is, whether the positive band of the pair is associated with the parallel band or with the perpendicular band. The sign of the factor, $\mu_{0 \mathrm{e}, t} \mu_{0 \mathrm{e}, / l}$, will be visuallized, for example, by assigning a coordinate system for a transition electric dipole, as illustrated in Figure 2. If the dipole directs from the octant I to VII (un-

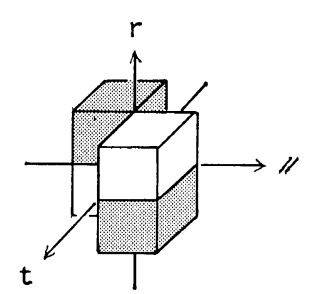

$\mu_{0 e, t} \mu_{0 e, 11}>0$

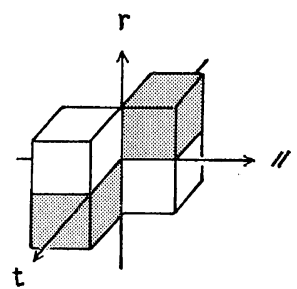

$\mu_{\cdot 0, t} \mu_{0 e, / 1}<0$

Figure 2. The coordinate system $(t, /, r)$ and directions of transition electric dipole.

hatched), or from the octant III to V (hatched), then $\mu_{0 \mathrm{e}, t} \mu_{0 \mathrm{e}, / /}>0$, and if it is in a different direction from the above (i.e., from II to VIII (hatched) or from IV to VI (unhatched)), then $\mu_{0 \mathrm{e}, t} \mu_{0 \mathrm{e}, \|}<0$. If we trace the dipole moment vectors toward their directions along the surface of circumscribed cylinder, then we follow a right-handed helical way for $\mu_{0 e, t} \mu_{0 e, / /}>0$, while we have a left-handed helical locus for $\mu_{0 e, t} \mu_{0 e, l l}$ $<0$. For $\mu_{0 \mathrm{e}, t} \mu_{0 \mathrm{e}, / /}>0$, the positive ellipticity band is associated with the parallel band, while for $\mu_{0 \mathrm{e}, t} \mu_{\mu_{\mathrm{e}}, /}<0$, it is associated with the perpendicular band.

The equation for the total rotatory oscillator strength term, eq 32, together with eq 23 , defines the sign of ellipticity bands more directly, that is, whether the positive band of a pair appears at the longer wavelength side or at the shorter wavelength side, for an electrically allowed transition. It is governed by the sign of sum of interaction potentials, $\sum_{\substack{k=1 \\ k+l: \text { odd }}}^{N} \sum_{\substack{l \neq k \\ \text { odd }}}^{N} V_{k 0 \mathrm{e}, l 0 \mathrm{e}}$, as well as by the direction of transition dipole, $\mu_{0 \mathrm{e}, t} \mu_{0 \mathrm{e}, \|}$, that is, by the sign of their product. A positive band appears at the longer wavelength side if the sign of the product is negative, while it is at the shorter wavelength side if the sign is positive.

\section{CIRCULAR DICHROISM OF THE $N-V_{1}$ TRANSITION OF POLYPEPTIDE}

Although we do not know any example of an extended polypeptide chain in an isolated form, we will calculate circular dichroism associated with the $N-V_{1}$ transition of peptide chromophores in such a chain. The $N-V_{1}$ transition 


\section{S. IKEDA}

is electrically allowed, and only the exciton contribution to rotatory power will be estimated. Geometry of an extended polypeptide chain was chosen from that of a polypeptide chain found in $\beta$-form poly(L-alanine), whose atomic positions were determined by Arnott, Dover, and Elliott. ${ }^{14}$

Direction of the transition electric dipole was assigned as that found in myristamide, ${ }^{15}$ and it was located at the center of gravity of transi- tion monopole charges of each peptide group, which were taken from Woody and Tinoco. ${ }^{16}$ The dipole and monopole parameters adapted to the extended polypeptide are listed in Tables III and IV. It is evident that the direction of the transition dipole gives $\mu_{0 \mathrm{e}, t} \mu_{0 \mathrm{e}, / /}>0$.

Calculations were performed on the Facom 230-60 of the Nagoya University Computation Center. The chain length, $N$, was varied from 2 to 20 . The interaction potential was evaluated

Table III. Geometric and electronic parameters of the $N-V_{1}$ chromophore in an extended polypeptide

\begin{tabular}{cccccc}
\hline$a, \AA$ & $b, \AA$ & $\lambda_{\mathrm{EO}}=\frac{\nu_{\mathrm{EO}}}{c}, \mathrm{~m} \mu$ & $\left|\mu_{0 \mathrm{e}}\right|, \mathrm{D}$ & $\frac{\mu_{0 \mathrm{e}, \mathrm{t}}}{\left|\mu_{0 \mathrm{e}}\right|}$ & $\frac{\mu_{0 \mathrm{e}, l l}}{\left|\mu_{0 \mathrm{e}}\right|}$ \\
\hline 0.403 & 3.445 & 190.00 & 3.050 & 0.7936 & 0.5648 \\
\hline
\end{tabular}

Table IV. Monopole parameters for the $N-V_{1}$ transition of an extended polypeptide

\begin{tabular}{cccrc}
\hline$\sigma$ & $\rho_{0 e, \sigma}, 10^{-10}$ e.s.u. & $x, \AA$ & $z, \AA$ & $y, \AA$ \\
\hline Peptide oxygen & -0.903 & -1.700 & 0.130 & -3.530 \\
Peptide carbon & -0.821 & -0.490 & -0.130 & -3.560 \\
Nitrogen & 1.724 & 0.330 & 0.130 & -2.560 \\
\hline
\end{tabular}

Table V. Spectral strengths for the two exciton levels of the $N-V_{1}$ transition $^{a}$

\begin{tabular}{|c|c|c|c|c|c|}
\hline$N$ & $\lambda_{\mathrm{E} K}=\frac{c}{\nu_{\mathrm{E} K}}, \mathrm{~m} \mu$ & $\Delta \widetilde{\nu}_{\mathrm{E}}, \mathrm{cm}^{-1 \mathrm{~b}}$ & $\frac{D_{\mathrm{E} K}}{N}, \mathrm{D}^{2}$ & $\frac{R_{\mathrm{EK}}}{N}, \mathrm{DBM}$ & $\frac{\sum\left(\nu_{\mathrm{E} K}-\nu_{\mathrm{EO}}\right) R_{\mathrm{E} K}}{N}, 10^{-45}$ c.g.s. ${ }^{\mathrm{c}}$ \\
\hline \multirow{2}{*}{2} & 194.01 & \multirow{2}{*}{2175} & 2.97 & 0.300 & \multirow{2}{*}{-0.202} \\
\hline & 186.15 & & 6.33 & -0.300 & \\
\hline \multirow{2}{*}{3} & 195.69 & \multirow{2}{*}{3075} & 3.06 & 0.283 & \multirow{2}{*}{-0.269} \\
\hline & 184.58 & & 6.24 & -0.283 & \\
\hline \multirow{2}{*}{4} & 196.58 & \multirow{2}{*}{3549} & 2.82 & 0.285 & \multirow{2}{*}{-0.307} \\
\hline & 183.76 & & 6.03 & -0.285 & \\
\hline \multirow{2}{*}{6} & 197.45 & \multirow{2}{*}{4010} & 2.74 & 0.277 & \multirow{2}{*}{-0.346} \\
\hline & 182.96 & & 5.86 & -0.277 & \\
\hline \multirow{2}{*}{10} & 198.08 & \multirow{2}{*}{4345} & 2.66 & 0.268 & \multirow{2}{*}{-0.379} \\
\hline & 182.39 & & 5.68 & -0.269 & \\
\hline \multirow{2}{*}{$\infty^{\mathrm{d}}$} & 198.11 & \multirow{2}{*}{4366} & 2.97 & 0.300 & \multirow{2}{*}{-0.405} \\
\hline & 182.34 & & 6.33 & -0.300 & \\
\hline
\end{tabular}

a $K$ refers to 1 or $N$ in eq 36 and represents the parallel or perpendicularly polarized exciton level.

b $\Delta \widetilde{\nu}_{E}=\left(\nu_{E N}-\nu_{E 1}\right) / c=\left(\nu_{E \perp}-\nu_{E} \| /\right) / c$.

c Eq 32. $\Sigma$ extends over $K$ from 1 to $N$.

d $\lambda_{\mathrm{E} K}$ 's were calculated by eq 38 . $\lambda_{\mathrm{E} K}$ and $\sum\left(\nu_{\mathrm{E} K}-\nu_{\mathrm{EO}}\right) R_{\mathrm{E} K} / N$ are for $N=20$. 
by the monopole approximation, eq 28 .

Spectral strengths associated with all the exciton levels were calculated by eq 29 and 30 , after solving eq 12 . It was found that all the interactions for odd $k+l$ values are attractive and the nearest neighbor interaction $\left(V_{10 \mathrm{e}, 20 \mathrm{e}}=\right.$ $-216.0 \times 10^{-15}$ c.g.s.) amounts to more than 20 times that of any other more distant interactions. As predicted by the nearest neighbor approximation, only two exciton levels have significant strengths of almost equal magnitude but of opposite sign, and their magnitudes are scarcely dependent on the chain length. The magnitudes of the two rotatory strengths are more than 20 times larger than those for the other levels. The parallel polarized level is at the longest wavelength side, having a positive rotatory strength, while the perpendicular level is at the shortest wavelength side. Spectral strengths associated with those two active levels are given in Table $\mathrm{V}$.

Circular dichroism arising from the two strong ellipticity bands was calculated by means of the

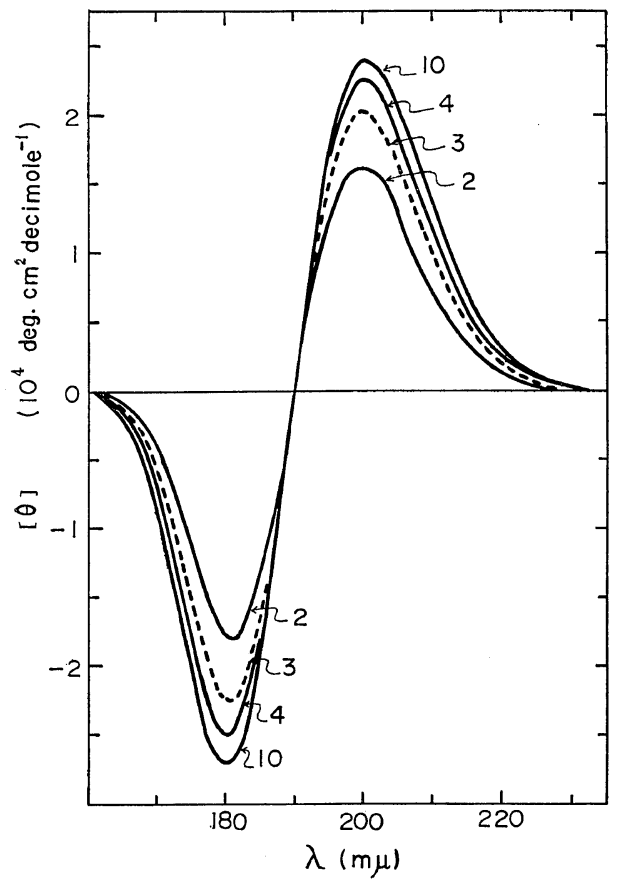

Figure 3. Oircular dichroism for the $N-V_{1}$ transition of an extended oligopeptide chain. Figures represent number of peptide groups in a chain. two-term equation of the form, eq 36 . The spectral shape was assumed to be Gaussian with a half-width, $3500 \mathrm{~cm}^{-1}$. Calculated spectra are shown in Figure 3. As the magnitude of residue ellipticity of the paired bands should be strongly dependent on the half-width, (and as the rotatory strength defines only the area for an ellipticity band,) Figure 3 must be regarded as showing a qualitative picture of conservative circular dichroism.

Circular dichroism spectra were also calculated by the expansion approximation, eq 23 together with eq 32 . The total rotatory oscillator strength term must vary with the chain length, as shown in Table $\mathrm{V}$, since it includes the interaction potentials explicitly. With the same spectral shape as used above, magnitudes of residue ellipticity were more than twice as large as those calculated by eq 36 , for large $N$ values. This would come from the approximate nature of eq 22 as well as from the question whether an identical spectral shape or half-width may be used for both eqs 36 and 23 .

In place of the monopole approximation, the interaction potential was also evaluated by the dipole approximation, eq 27 . The nearest neighbor interaction $\left(V_{10 \mathrm{e}, 20 \mathrm{e}}=-26.8 \times 10^{-15}\right.$ c.g.s. $)$ was slightly larger in magnitude, but, as expected from the nearest neighbor approximation, the exciton coefficients were scarcely influenced by the nature of approximation for interaction. Thus the magnitude of spectral strengths remains unchanged, although their location shifts.

Woody ${ }^{17}$ has drawn distribution diagram of spectral strengths and tabulated their location and magnitude for the the $\beta$-structure of a polypeptide. Some of his results (on the singlestranded pleated sheet structures) could be compared with the present calculations.

\section{APPENDIX. DERIVATION OF EQ 24}

Multiplication of eq 11 by eq 14 and summation over $K$ lead to

$$
\begin{aligned}
& \sum_{K=1}^{N}\left(\nu_{\mathrm{E} K}-\nu_{\mathrm{EO}}\right) R_{\mathrm{E} K} \\
& =(1 / h) \sum_{K=1}^{N} \sum_{k=1}^{N} \sum_{l \neq k}^{N} C_{k \mathrm{e} K} C_{l \mathrm{e} K} V_{l 0 \mathrm{e}, l 0 \mathrm{e}} \\
& \quad \times \sum_{\kappa=1}^{N} \sum_{\lambda=1}^{N} C_{\kappa \mathrm{e} K} C_{\lambda \mathrm{e} K} F_{\kappa, \lambda}
\end{aligned}
$$




\section{S. IKEDA}

where

$$
\begin{aligned}
F_{\kappa, \lambda}= & \operatorname{Im}\left(\boldsymbol{\mu}_{\kappa 0 \mathrm{e}} \cdot \mathbf{m}_{\lambda \mathrm{eO}}\right) \\
& -\left(\pi \nu_{\mathrm{EO}} / 2 c\right)\left(\left(\mathbf{R}_{\lambda}-\mathbf{R}_{\kappa}\right) \cdot\left[\boldsymbol{\mu}_{\kappa 0 \mathrm{e}} \times \boldsymbol{\mu}_{\lambda 0 \mathrm{e}}\right]\right)
\end{aligned}
$$

Let us evaluate

$$
\begin{aligned}
Y= & \sum_{K} \sum_{L}\left(\sum_{k} \sum_{l} C_{k \ominus K} C_{l \mathrm{e} L} V_{k 0 \mathrm{e}, l 0 \mathrm{e}}\right) \\
& \times\left(\sum_{\kappa} \sum_{\lambda} C_{\kappa \mathrm{e} K} C_{\lambda \mathrm{e} L} E_{\kappa, \lambda}\right)
\end{aligned}
$$

in two ways. First procedure is to use eq 10 and then $V_{k 0 \mathrm{e}, k 0 \mathrm{e}}=0$.

$$
\begin{aligned}
Y= & \sum_{k} \sum_{\kappa}\left(\sum C_{k \mathrm{e} K} C_{\kappa \mathrm{e} K}\right) \\
& \times \sum_{l} \sum_{\lambda}\left(\sum_{L} C_{l \mathrm{e} L} C_{\lambda \mathrm{e} L}\right) V_{k 0 \mathrm{e}, l 0 \mathrm{e}} F_{\kappa, \lambda} \\
= & \sum_{k} \sum_{l} V_{l k 0 \mathrm{e}, l 0 \mathrm{e}} F_{k, l}=\sum_{k=1} \sum_{l \neq k}^{\prime} V_{k 0 \mathrm{e}, l 0 \mathrm{e}} F_{k, l}
\end{aligned}
$$

Alternately, $Y$ is divided into two, $Y_{K K}$ and $Y_{K L}$, and calculated separately. $Y_{K K}$ reduces to the left-hand side of eq $\mathrm{Al}$ and $Y_{K L}$, vanishes, as shown below.

$$
\begin{aligned}
Y_{K K} & =\sum_{K} \sum_{k} \sum_{l} C_{k \mathrm{e} K} C_{l \mathrm{e} K} V_{k 0 \mathrm{e}, l 0 \mathrm{e}} \sum_{k} \sum_{\lambda} C_{\kappa \Theta K} C_{\lambda \mathrm{e} K} F_{\kappa, \lambda} \\
& =\sum_{K} \sum_{k} \sum_{l}^{\prime} C_{k \mathrm{e} K} C_{l \mathrm{e} K} V_{k 0 \mathrm{e}} \sum_{\kappa} \sum_{\lambda} C_{\kappa \mathrm{e} K} C_{\lambda \mathrm{e} K} F_{\kappa, \lambda}, \\
& =h \sum_{K}\left(\nu_{\mathrm{E} K}-\nu_{\mathrm{EO}}\right) R_{\mathrm{E} K}
\end{aligned}
$$

and

$$
\begin{aligned}
Y_{K L}= & \sum_{K} \sum_{L \neq K}^{\prime}\left(\sum_{k} \sum_{l} C_{k \mathrm{e} K} C_{l \mathrm{e} K} C_{k 0 \mathrm{e}, l 0 \mathrm{e}}\right) \\
& \times \sum_{k} \sum_{\lambda} C_{\kappa \mathrm{e} K} C_{\lambda \mathrm{e} L} F_{\kappa \lambda}=0
\end{aligned}
$$

since

$$
\begin{aligned}
& \sum_{l} \sum_{l} C_{k \mathrm{e} K} C_{l \mathrm{e} L} V_{k 0 \mathrm{e}, l 0 \mathrm{e}} \\
& \quad=\sum_{k} \sum_{l} C_{k \mathrm{e} K} C_{l \mathrm{e} L} \int \psi_{l \mathrm{e}}^{0} V_{k, l} \psi_{l \mathrm{e}}^{0} \mathrm{~d} \tau \\
& \quad=2 \int \phi_{\mathrm{E} K}^{0}\left(H-\sum H_{k)}\right) \psi_{\mathrm{E} L}^{0} \mathrm{~d} \tau
\end{aligned}
$$

$$
\begin{aligned}
& =2\left(E_{\mathrm{E} L}^{1}-E_{\mathrm{e}}^{0}\right) \int \psi_{\mathrm{E} K}^{0} \psi_{\mathrm{E} L}^{0} \mathrm{~d} \tau \\
& =0
\end{aligned}
$$

Combination of eq A3 with A4 is identical with eq 24. Equation 24 was first derived by Moffitt, Fitts, and Kirkwood. ${ }^{2}$

\section{REFERENCES}

1. W. Moffitt, J. Chem. Phys., 25, 467 (1956).

2. W. Moffitt, D. D. Fitts, and J. G. Kirkwood, Proc. Nat. Acad. Sci., U. S., 43, 723 (1957).

3. I. Tinoco, Jr., Advan. Chem. Phys., 4, 113 (1962).

4. I. Tinoco, Jr., R.W. Woody, and D.F. Bradley, J. Chem. Phys., 38, 1317 (1963).

5. I. Tinoco, Jr., J. Amer. Chem. Soc., 86, 297 (1964).

6. I. Tinoco, Jr., J. chim. physique, 65, 91 (1968).

7. T. Ando, Prog. Theor. Phys., 40, 471 (1968).

8. F. M. Loxsom, J. Chem. Phys., 51, 4899 (1969).

9. C. W. Deutsche, ibid., 52, 3703 (1970).

10. D. F. Bradley, I. Tinoco, Jr., and R. W. Woody, Biopolymers, 1, 239 (1963).

11. D. F. Bradley, S. Lifson and B. Honig, in "Electronic Aspects in Biochemistry," B. Pullman, Ed., Academic Press., London, p 77.

12. W. Moffitt and A. Moscowitz, J. Chem. Phys., 30, 648 (1959).

13. N. Go, in "Quantum Biology," Vol. II, the Biophysical Society of Japan, Ed., 1965, Yoshioka Shoten, Kyoto, (in Japanese), p 257.

14. S. Arnott, S. D. Dover, and A. Elliott, J. Mol. Biol., 30, 201 (1967).

15. D. L. Peterson and W. T. Simpson, J. Amer. Chem. Soc., 79, 2375 (1957).

16. R. W. Woody and I. Tinoco, Jr., J. Chem. Phys., 46, 4927 (1967).

17. R. W. Woody, Biopolymers, 8, 669 (1969). 